\title{
A case of Zellweger syndrome accompanied by hypertrophic
} cardiomyopathy

\author{
Yusuf Kale ${ }^{1 *}$, Istemi Han Celik ${ }^{2}$, Ferit Kulali ${ }^{2}$, Osman Yilmaz ${ }^{2}$, Ahmet Yagmur Bas ${ }^{2}$, \\ Nihal Demirel ${ }^{2}$
}

\begin{abstract}
Peroxisomal biogenesis disorders are a group of genetically and clinically heterogenous disorders which affect very-long chain fatty acid metabolism. Zellweger syndrome (ZS) is a rare, congenital disorder characterized by multisystem involvement including central nervous system, skeletal system, liver, kidney and eyes, due to absence of peroxisomes in the cells. Hypertrophic cardiomyopathy (HCM) is defined as septal or posterior wall thickness that is more than two standard deviations above the mean normal thickness measured by echocardiography. Here we present a newborn with Zellweger syndrome and hypertrophic cardiomyopathy.
\end{abstract}

Key words: Hypertrophic Cardiomyopathy, Zellweger Syndrome, Newborn

\section{Introduction}

Zellweger syndrome (OMIM 214100) is the prototype disorder of a group of peroxisomal biogenesis disorders. Zellweger Syndrome (ZS) is a fatal autosomal-recessive hereditary disease characterized by the reduction or absence of peroxisomes in the cells of the liver, kidneys and brain. The absence of peroxisomes results in impairment of many metabolic pathways, especially beta-oxidation of very long chain fatty acids (VLCFA). In the newborn period, effected children are hypotonic, feed poorly and have distinctive facies, seizures, hepatic dysfunction and renal cysts $(1,2)$.

HCM is defined as septal or posterior wall thickness that is more than two standard deviations above the mean normal thickness measured by echocardiography (3). In the newborn or the fetus, transient hypertrophic cardiomyopathy is commonly attributed to certain maternal metabolic disorders (in particular, diabetes mellitus) or to antenatal or postnatal exposure to steroids. In addition, hypertrophic cardiomyopathy has been described in association with genetic syndromes and, in rare cases, as a primary lesion associated with other congenital heart defects (4). In this report, we present a case of ZS accompanied by hypertrophic cardiomyopath.

\section{Case}

A 3110-g female infant was born at 40 weeks' gestation to a 26- year-old gravida 2, para 2, by cesarean delivery. Apgar scores were 7 and 8 at 1 minute and 5minutes, respectively, and no resuscitation was required. Her head circumference, weight and length were $35 \mathrm{~cm}$ (50th centile), $3110 \mathrm{gr}$ (25-50th centile), and $51 \mathrm{~cm}$ (50-75th centile), respectively. Pregnancy was uneventful and fetal growth was normal on follow-up. She was the second offspring of healthy parents with no consanguinity. The first child had died of ZS at 4 months of age. After birth, she was noted to be floppy and required oxygen supplementation and was transferred to the neonatal intensive care unit.

On physical examination the infant had atypical facial appearance with epicantal folds, high forehead and large anterior fontanel $(3 \mathrm{X} 4 \mathrm{~cm})$ (Figure1). She had mild tachypnea without retractions, hypotonia and diminished neonatal reflexes. The liver is palpable 2 $\mathrm{cm}$. An ultrasound examination of kidneys showed increased echogenicity and cortical cysts. Cranial ultrasound was normal. Karyotype demonstrated 46,XX.

On 9th day, a grade 2/6 systolic murmur was noted. Mild cardiomegaly and normal pulmonary vascular markings were present on the chest radiograph. 
Figure 1: Facial appearance
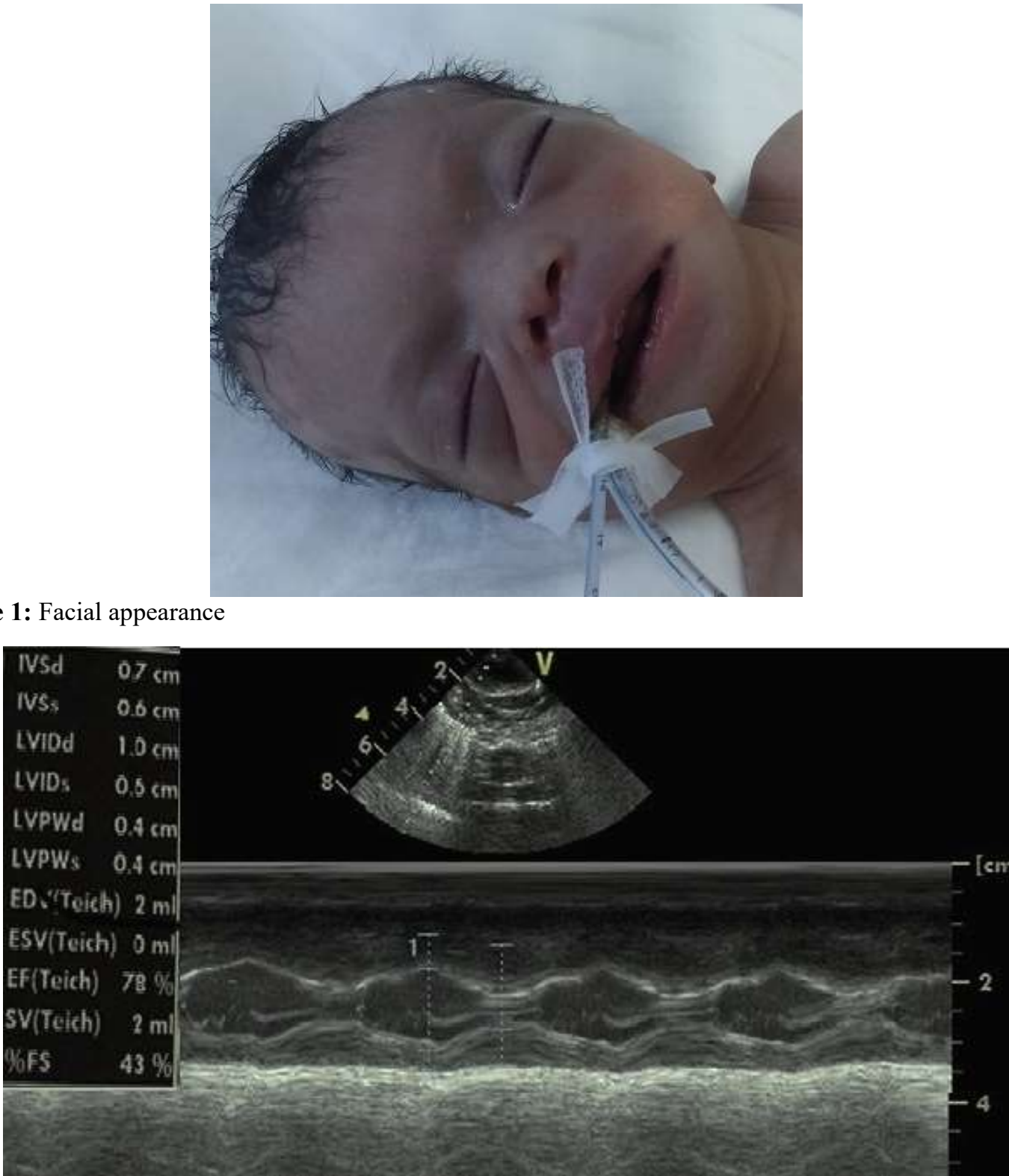

Figure 2: Echocardiography showing interventricular septum thickening (IVSd Z $0.7 \mathrm{~cm})$

Table 1: Serum levels of VLCFA and phytanic acid

\begin{tabular}{lcc} 
VLCFAs & Patients & Normal Values \\
\hline $\mathrm{C} 26: 0(\mu \mathrm{mol} / \mathrm{L})$ & 10,10 & $0,6-1,3$ \\
$\mathrm{C} 24: 0(\mu \mathrm{mol} / \mathrm{L})$ & 46,43 & $37,4-79,4$ \\
$\mathrm{C} 22: 0(\mu \mathrm{mol} / \mathrm{L})$ & 35,61 & $41,1-90,3$ \\
$\mathrm{C} 24: \mathrm{C} 22$ & 1,3 & $0,68-1,008$ \\
$\mathrm{C} 26: \mathrm{C} 22$ & 0,28 & $0,011-0,026$ \\
Phytanic acid $(\mu \mathrm{mol} / \mathrm{L})$ & 1,31 & $<5,28$
\end{tabular}

Echocardiographic evaluation showed marked thickening of the interventricular septum (IVS) (0.7 $\mathrm{cm}$ ) (Figure 2). The family history was negative for hypertrophic cardiomyopathy or sudden death. A maternal drug history was unremarkable and oral glucose tolerance test was normal. The maternal glycated hemoglobin (HbAlc) level was $4.6 \%$.

\footnotetext{
Medical Science and Discovery, 2016; 3(5):242-4
}

Laboratory tests upon admission including complete blood count, peripheral blood smear, C-reactive protein, serum glucose, electrolytes, renal and liver function tests were unremarkable. Tandem mass spectroscopy analysis was normal. The baby was completely asymptomatic all the time. 
The infant remained hypotonic with no sucking reflex and was commenced on orogastric tube feeding. Oxygen requirement resolved within 10 days and supplemental oxygen was discontinued. Hepatomegaly was noted on day 12 , and bilirubin, alanine aminotransferase (ALT) and aspartate aminotransferase (AST) started to rise. Laboratory investigations revealed ALT of $307 \mathrm{IU} / \mathrm{L}, \mathrm{AST}$ of 750 $\mathrm{IU} / \mathrm{L}$, total bilirubin of $6.4 \mathrm{mg} / \mathrm{dL}$ and direct bilirubin of $3.4 \mathrm{mg} / \mathrm{dL}$.

Phenotypic features, generalized hypotonia, renal cortical cysts and elevated liver transaminases suggested peroxisomal disorders, and VLCFA analysis was ordered which was compatible with Zellweger syndrome (Table 1). PEX1 gene analysis was performed, which showed a previously reported mutation: homozygous p.V100Gfs*32. On day 27 of life she developed aspiration pneumonia and died on day 29 of life following worsening of his respiratory status.

\section{Discussion}

Zellweger syndrome is a fatal autosomal recessive disease caused by an absence of functional peroxisomes. Impaired metabolism results in the accumulation of toxic metabolites, which give damage to developing neurons (5). Severe psychomotor retardation, severe hypotonia at birth, dysmorphic facial features and liver dysfunction are the hallmarks of this disease. Central nervous system abnormalities encountered in this disease include cortical dysplasia, neuronal migration defects and dysmyelination. Affected infants usually have small renal cortical cysts. Patients usually die within a few months after birth (5).

Prenatal diagnosis is possible via enzyme assays using chorion villous samples or amniocytes or by analysis of VLCFA in amniotic fluid. If the genetic defect has been identified in the index patient, DNA analysis can be performed (2).

Zellweger syndrome may be accompanied by cardiac anomalies, which include ventricular septal defect, patent ductus arteriosus and aortic arch anomalies (6). Our patient only had hypertrophic cardiomyopathy diagnosed by echocardiography. Hypertrophic cardiomyopathy in infancy has been described as a result of exposure to maternal diabetes or to corticosteroids.
In addition, hypertrophic cardiomyopathy has been described in association with perinatal asphyxia, metabolic diseases, in utero ritodrine exposure, Noonan syndrome, systemic hypertension, and familial HCM (7). In our case, prenatal history was negative for maternal risk factors. Oral glucose tolerance test performed during pregnancy and the postpartum HbAlc level were in the normal range in our patient. Although rare, all newborns with Zellweger syndrome should be evaluated by echocardiography for accompanying cardiac defects.

Conflict of Interest: The authors declare no potential conflicts of interest with respect to the research, authorship, and/or publication of this article.

Ethical issues: All Authors declare that Originality of research/article etc... and ethical approval of research, and responsibilities of research against local ethics commission are under the Authors responsibilities. The study was completed due to defined rules by the Local Ethics Commission guidelines and audits.

Acknowledgement: This research received no specific grant from any funding agency, commercial or not-for- profit sectors.

\section{References}

1. Wanders, R. J. A. Metabolic and molecular basis of peroxisomal disorders: a review. Am. J. Med. Genet 2004; 126: 355-75.

2. Gould SJ, Raymond GV, Valle D. The peroxisome biogenesis disorders. In: Scriver CR, Beaudet AL, Valle D, Sly WS (eds) The metabolic \& molecular bases of inherited disease. New York: McGraw-Hill, 2001.p. 3181217.

3. $\mathrm{Ng}$ PC. The effectiveness and side effects of dexamethasone in preterm infants with bronchopulmonary dysplasia. Arch Dis Child 1993; 68: 330-36.

4. Ben-Shacher G, Moller JH, Castaneda-Zuniga W, Edwards JE. Signs of membranous subaortic stenosis appearing after correction of persistent common atrioventricular canal. Am J Cardiol 1981; 48: 340-4.

5. Grayer J. Recognition of Zellweger syndrome in infancy. Adv Neonatal Care. 2005; 5: 5-13.

6. Kelley RI, Datta NS, Dobyns WB, Hajra AK, Moser AB, Noetzel MJ, et al. Neonatal adrenoleukodystrophy: New cases, biochemical studies, and differentiation from Zellweger and related peroxisomal polydystrophy sydnromes. Am J Med Genet 1986; 23: 869-901.

7. Badertscher A, Bauersfeld U, Arbenz U, Baumgartner MR, Schinzel A, Balmer C. Cardiomyopathy in newborns and infants: a broad spectrum of aetiologies and poor prognosis. Acta Paediatr 2008; 97: 1523-8.

Copyright (C) 2014 The Author(s); This is an open-access article distributed under the terms of the Creative Commons Attribution License (http://creativecommons.org/licenses/by/4.0), which permits unrestricted use, distribution, and reproduction in any medium, provided the original work is properly cited. All Rights reserved by international journal of Medical Science and Discovery. 